\title{
Philosophiques
}

\section{Mimèsis et catharsis : de la représentation à la dénégation du réel chez Aristote, Artaud et Brecht}

\section{Alain Bernard Marchand}

Volume 15, numéro 1, printemps 1988

URI : https://id.erudit.org/iderudit/027038ar

DOI : https://doi.org/10.7202/027038ar

Aller au sommaire du numéro

Éditeur(s)

Société de philosophie du Québec

ISSN

0316-2923 (imprimé)

1492-1391 (numérique)

Découvrir la revue

\section{Citer cet article}

Marchand, A. B. (1988). Mimèsis et catharsis : de la représentation à la dénégation du réel chez Aristote, Artaud et Brecht. Philosophiques, 15(1), 108-127. https://doi.org/10.7202/027038ar
Résumé de l'article

La présente étude propose une relecture de trois théoriciens dont les investigations continuent à servir de pierre angulaire à la théâtrologie : celles d'Aristote dont La Poétique, outre le fait qu'elle consacre le théâtre occidental, sert de fondement à l'esthétique dramatique et celles, plus récentes, d'Antonin Artaud et de Bertolt Brecht qui, bien qu'ils aient réfuté radicalement les théories aristoté- liciennes, ne se sont pas moins distingués l'un de l'autre pour donner les deux grandes voies que l'on sait à la réflexion dramaturgique contemporaine. Ces spéculations seront envisagées sous l'éclairage décisif de la mimèsis et de la catharsis qui ne se posent pas chez eux comme des notions distinctes, mais comme l'avers et l'envers d'un même travail sur un réel à chaque fois redéfini, qu'il s'agisse de l'obéissance aristotélicienne à une stricte logique narrative, de l'utopique déculturation artaudienne ou de l'engagement brechtien. 


\title{
ARTICLES \\ MIMÈSIS ET CATHARSIS : DE LA REPRÉSENTATION A LA DÉNÉGATION DU RÉEL CHEZ ARISTOTE, ARTAUD ET BRECHT
}

\author{
par Alain Bernard Marchand
}

RÉSUMÉ. La présente étude propose une relecture de trois théoriciens dont les investigations continuent à servir de pierre angulaire à la théâtrologie : celles d'Aristote dont La Poétique, outre le fait qu'elle consacre le théâtre occidental, sert de fondement à l'esthétique dramatique et celles, plus récentes, d'Antonin Artaud et de Bertolt Brecht qui, bien qu'ils aient réfuté radicalement les théories aristotéliciennes, ne se sont pas moins distingués l'un de l'autre pour donner les deux grandes voies que l'on sait à la réflexion dramaturgique contemporaine. Ces spéculations seront envisagées sous l'éclairage décisif de la mimèsis et de la catharsis qui ne se posent pas chez eux comme des notions distinctes, mais comme l'avers et l'envers d'un même travail sur un réel à chaque fois redéfini, qu'il s'agisse de l'obéissance aristotélicienne à une stricte logique narrative, de l'utopique déculturation artaudienne ou de l'engagement brechtien.

ABSTRACT. The author proposes a review of three major theoreticians whose works are considered to be the corner-stones of theatrical studies: Aristotle, whose Poetics, the first reflexion on Occidental theatre, also serve as the foundation of the aesthetics of drama, and, closer to us, Antonin Artaud and Bertolt Brecht who, although unanimous in rejecting the Aristotelian theory, have quite different views on contemporary theatre. Their definitions are analyzed in the light of mimesis and catharsis, which are not taken here as separate notions, but rather as a mean of grasping the fluctuant outlines of the concept of "reality", be it Aristotle's obedience to a strict narrative structure, Artaud's denial of culture or Brecht's political commitment. 
"Pourquoi trouvons-nous de l'intérêt à représenter
sur scène le meurtre et la folie, alors que, dans la
vie réelle, nous jugeons et nous enfermons les
meurtriers et les fous? Pourquoi, surtout,
employons-nous des acteurs professionnels, et les
payons-nous de cachets élevés, au lieu de permettre
au protagoniste de se représenter lui-même?
C'est sans doute parce que nous ne supporterions
pas de nous trouver en face de véritable détresse,
de véritable folie, de véritables éclats de violence.
Nous avons donc inventé une forme supportable
d'expression, le théâtre, dans laquelle le même
processus, qui nous effraie normalement, nous
donne maintenant du plaisir. Nous avons maîtrisé
les liens, dompté les bêtes sauvages et nous nous
sommes délivrés de notre faute. C'est pour cela
qu'il est permis à l'acteur d'apparaître sur scène,
libéré de ses chaînes, et de nous libérer par là
même ».

Moreno, Psychotbérapie de groupe et psychodrame

A. QUELQUES POINTS DE REPÈRE :

\section{LA RELATION MIMĖSIS-CATHARSIS}

L'énigme de la catharsis tient en grande partie à son indétermination. Rien de plus énigmatique, pour la réflexion théâtrale, que l'idée de catharsis telle qu'elle traverse les différents discours qui l'ont interrogée comme le point de jonction de la représentation et de la résolution d'une certaine (dé)charge émotive. Déjà l'étymologie prête à équivoque, et le mot purgation par lequel les latinistes ont traduit le grec katharsis renvoie à la fois à une épuration physiologique et morale: corps et âme mêmement débarrassés de traces impures, c'est-à-dire interdites au bon rendement de la société. On serait donc tenté de définir la catharsis comme la libération d'un en trop dont il faut se purger pour retrouver la commune mesure. Au théâtre, il s'agirait d'une perturbation simulée, rendue sensible grâce à la scène, qui distord impunément la Loi de la Cité pour en chasser toutes les pulsions indésirables : la catharsis est, en quelque sorte, un rite purgatif qui consiste à représenter et à exorciser les angoisses et les désirs d'un peuple. C'est ce que Hitchcock le cinéaste appellera plus tard des 


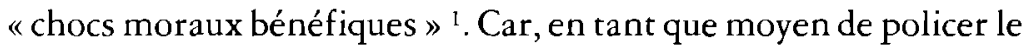
refoulé collectif, la catharsis, si elle se trouve assimilée à la fixation d'un en trop, opère corrélativement le retour à une norme prescrite par la Loi sociale et, pourrait-on ajouter, corporelle si l'on prend la référence médicale à la lettre : le corps agissant de l'acteur et agi du spectateur comme manifestation du comportement social et comme métaphore de l'idéologie en jeu. Récupération efficace des pulsions indomptées de la Cité, la catharsis est somme toute une façon institutionnalisée de mettre en scène l'interdit et de s'en libérer en le vivant «par procuration $»^{2}$ : corps, âme et société ainsi purgés d'une démesure jugée incivique. Telle est, si l'on peut dire, la conception politique de la catharsis.

Une première évidence s'impose d'emblée lorsqu'on envisage la catharsis d'un point de vue fonctionnel : elle se révèle indissociable de l'acte de représenter et, par extension, d'une spécificité théâtrale que l'on n'a cessé de redéfinir depuis Aristote. En effet, si elle fonctionne comme opération purgative, c'est qu'elle (re)produit et dénie en même temps une certaine acceptation du réel qui se décompose au théâtre en un monde figuré et en un réel scénique concret. Impossible, dès lors, de s'interroger sur la catharsis sans faire appel à la notion un peu plus explicite de mimèsis. Celle-ci se résume essentiellement à l'acte de représenter, mais encore faut-il ne pas se méprendre sur le sens de la représentation: il ne s'agit certes pas de la plate imitation d'un réel, si fidèle soit-elle, mais de sa (re)formulation. En un mot, la mimèsis consiste à transformer l'ordre du réel en un tout autre ordre qui le déréalise pour «rend(re) possible le retour du refoulé sous sa forme niée» ${ }^{3}$.

1. François Truffaut, Hitchcock/Truffaut, (Turin : Éd. Ramsay, 1983.) p. 168. Hitchcock propose ici une définition de la notion de catharsis en l'associant au domaine du cinéma : "Je suis prêt à procurer des chocs moraux bénéfiques. La civilisation est devenue si protectrice qu'il ne nous est plus pcssible de nous procurer instinctivement la chair de poule. C'est pourquoi, afin de nous dégourdir et récupérer notre équilibre moral, il faut susciter ce choc artificiellement. Le cinéma me paraît être le meilleur moyen d'atteindre ce résultat $\gg$.

2. Nous empruntons cette expression très parlante à Anne UBERSFELD qui, cherchant à définir le plaisir théâtral chez Brecht (et en général), observe qu'il "tient pour une part considérable à cette construction visible et tangible d'un fantasme que l'on peut vivre par procuration sans être tenu de le vivre pour soi, dangereusement ". Lire le théatre, (Paris : Éd. Sociales, 1978.) p. 57.

3. O Mannoni, Clefs pour l'imaginaire ou l'autre scène, (Paris : Éd. du Seuil, 1969.) p. 166. 
Roselyne Dupont-Roc et Jean Lallot, dans leur récente traduction de La Poétique d'A ristote, assimilent la mimèsis « (au) mouvement même qui partant d'objets préexistants aboutit à un artefact poétique et, précisent-ils, l'art poétique selon Aristote est l'art de ce passage » ${ }^{4}$. Il ne s'agit donc pas de présenter le réel, d'en imiter les formes plausibles, de le mettre sous les yeux du spectateur, mais de le re-présenter (du lat. reproesentare "rendre présent», de proesens «présent»), c'est-à-dire de le signifier à distance intentionnellement. «L'activité mimétique (...), ajoutent R. DupontRoc et J. Lallot, établit entre les deux objets, modèle et copie, une relation complexe ; elle implique à la fois ressemblance et différence, identification et transformation, d'un seul et même mouvement ${ }^{5}$. Il semblerait, à lire Aristote, que la mimèsis soit une première tentative de définir la fiction comme distance nécessaire par rapport au réel.

D'ailleurs, selon la formule de Anne Ubersfeld, «ce qui est imité (au théâtre) n'est pas le monde, mais le monde repensé selon la fiction et dans le cadre d'une culture et d'un code ${ }^{6}$. C'est dire la nature singulière du réel auquel fait signe toute activité mimétique et la portée sociale de l'opération cathartique qui en résulte. Dès lors que la relation mimèsis-catharsis se présente comme un double mouvement d'inscription et de déni du réel dans l'espace du jeu, le malaise cathartique, tel qu'on l'a souvent appelé, en vient à s'associer à un certain plaisir esthétique. Plaisir ambigu, certes, qui est peut-être celui de s'investir sans danger dans un lieu à l'abri du réel. Le théâtre serait ainsi une entreprise libératrice qui vise à déréaliser, voire à transcender l'ordre du réel. C'est dans cette optique que nous proposons, dans les pages qui suivent, une relecture de trois théoriciens dont les investigations continuent à servir de pierre angulaire à la théâtrologie : celles d'Aristote dont La Poétique, outre le fait qu'elle consacre le théâtre occidental, sert de fondement à l'esthétique dramatique et celles, plus récentes, d'Antonin Artaud et de Bertolt Brecht qui, bien qu'ils aient réfuté radicalement les théories aristotéliciennes, ne se sont pas moins

\footnotetext{
4. ARISTOTE (traduction et commentaires de Roselyne Dupont-Roc et Jean Lallot), $L a$ Poétique, (Paris : Éd. du Seuil, 1980.) p. 20.

5. Ibid., p. 157

6. A. Ubersfeld, L'école du spectateur, (Paris : Éd. Sociales, 1981.) p. 67.
} 
distingués l'un de l'autre pour donner les deux grandes voies que l'on sait à la réflexion dramaturgique contemporaine.

\section{B. ARISTOTE ET L'ÉMOTION QUINTESSENCIÉE}

«Ficrion is about unreal happiness and unhappiness, but these in their actuality ".

J.M. Redfield, Nature and Culture in the Iliad

Toute interrogation sur la catharsis consiste d'abord à faire parler le silence d'Aristote. La Poétique, à cet égard, n'est en effet guère parlante et, sauf pour quelques remarques sur la catharsis musicale dans le VIII livre de La Politique et la promesse d'y donner suite dans un complément de La Poétique qui nous est inconnu, l'explication aristotélicienne reste incomplète sinon énigmatique comme nous avons tôt fait de le signaler. Force nous est donc de spéculer sur le sens de cette purgation équivoque à partir de l'information que nous avons en main. Rappelons, avec Louis Moulinier, le caractère orgiastique de la flûte que souligne Aristote dans La Politique, si intensément amorale "qu'il faut se servir d'elle dans les circonstances où le spectacle peut produire la purification plutôt que l'enseignement ${ }^{7}{ }^{7}$. Moulinier conclut que la catharsis, définie comme purification, est en quelque sorte un phénomène « homéopathique ${ }^{8}$ : guérir l'excès par l'excès, l'éliminer en le montrant, donc l'intégrer à une pratique sociale vaguement religieuse. Barthes, d'un point de vue historique, a mis en doute l'utilité d'une telle restitution puisque l'on «ne (peut) avoir une idée du contexte, à la fois mystique et médical, si l'on peut dire, qui donne probablement son vrai sens à la notion de catharsis dramatique " 9. Néanmoins, il nous semble primordial, et en cela nous rejoignons les commentaires pénétrants de $\mathrm{R}$. Dupont-Roc et $\mathrm{J}$. Lallot, de restituer l'idée de catharsis dans une perspective qui

7. Cité par Louis Moulinier, Le pur et l'impur dans la pensée des Grecs, (Paris : Éd. Klincksieck, 1952.) Pp. 411-412.

8. Ibid., p. 417.

9. Roland BARTHES, «Le théâtre grec », in L'obvie et l'obtus, (Paris : Éd. du Seuil, 1982.) p. 71 . 
relève plus évidemment de la poétique dont l'objet premier est de réfléchir sur la régularisation d'un discours. C'est dire qu'il importe de dégager la spécificité théâtrale de la catharsis, s'il en est une, en regard de l'activité mimétique dont elle est dépendante comme le laisse entendre Aristote en soulignant que si la frayeur et la pitié naissent parfois de l'acte de montrer, donc des moyens propres au spectacle, "elles peuvent naître aussi du système des faits luimême (et) c'est là, ajoute-t-il impérieusement, le procédé qui tient le premier rang et révèle le meilleur poète ${ }^{10}$. Du reste, comme le remarque Paul Ricœur, Aristote néglige l'aspect de la mise en scène parce que son attitude est davantage celle d'un lecteur de théâtre que d'un spectateur. «(...) La Poétique, écrit-il, ne marque aucun intérêt explicite pour la communication de l'ouvre au public. Elle laisse même percer de place en place une réelle impatience à l'égard des contraintes liées à l'institution des concours (51 a 7) et plus encore à l'égard du mauvais goût du public ordinaire (chapitre XXV). La réception de l'œuvre n'est donc pas une catégorie majeure de La Poétique. Celle-ci est un traité relatif à la composition, sans presque aucun égard pour celui qui la reçoit » ${ }^{1}$. Il appert que la réalisation de la catharsis, selon l'idéal de $L a$ Poétique, tient à ce qu'on accorde la primauté à l'organisation du discours théâtral : le dire régissant le montrer de façon à ordonner un réel qui produise un effet particulier sur le spectateur.

Si le dire engendre principalement la mimèsis au théâtre, fabriquant l'illusion d'un réel possible, il faut bien comprendre ce qu'Aristote entend par là : il ne s'agit ni d'une invention fortuite ni d'une coulée « naturelle » de la chose racontée. Il s'agit plutôt d'une organisation de l'histoire, presque toujours préexistante, en un «système de faits » ${ }^{12}$, c'est-à-dire un arrangement logico-fonctionnel qui a le pouvoir de persuader le public car, selon Aristote, «le spectateur ne peut être affecté que si l'auteur réussit à le faire croire à ce qui est représenté » ${ }^{13}$. Cette volonté de privilégier nettement la disposition de la fable (plutôt que la fable elle-même) et d'expliquer la vraisemblance selon l'idéal de cohérence postulé

10. ARISTOTE, op. cit., p. 81.

11. Paul Ricceur, Temps et récit I, (Paris : Éd. du Seuil, 1983.) p. 80.

12. ARISTOTE, op. cit., p. 55.

13. Ibid., p. 399. 
par Aristote marque la lecture de P. Ricœur qui suit en cela les commentaires déjà fort révélateurs de R. Dupont-Roc et J. Lallot. "Ce serait un trait de la mimèsis, remarque-t-il, de viser dans le muthos non son caractère de fable, mais son caractère de cohérence. Son «faire » serait d'emblée un «faire» universalisant. Tout le problème du Versteben narratif est ici contenu en germe. Composer l'intrigue, c'est déjà faire surgir l'intelligible de l'accidentel, l'universel du singulier, le nécessaire ou le vraisemblable de l'épisodique ${ }^{14}$. On voit, dès lors, que la mimèsis aristotélicienne est avant tout affaire de langage: le langage théâtral, s'il donne l'illusion de la réalité, arrive surtout à ordonner le « réel " plutôt qu'à l'exprimer, et cela toujours en fonction d'une intentionnalité qui définit dans ses fondements le projet théâtral.

La persuasion, dans cette perspective, est le produit d'un agencement systématique et relève de l'organisation de la logique narrative. C'est dire que l'histoire n'avance pas (ne s'invente pas) inopinément ni gratuitement, mais qu'elle progresse selon une causalité qui la mène à produire certains effets voulus : l'histoire, autrement dit, est prise dans une structure qui assure son efficacité. Le «réel», on le voit déjà, n'est plus une simple expression innocente du monde, soit sa transcription dans l'œuvre, mais le résultat d'un ordonnancement qui le suscite. C'est là même la définition que donne Aristote de la fiction : du réel à l'histoire, il y a (re)structuration, voire (re)formulation du réel qui le fait passer du " particulier » au " général » ${ }^{15}$, du cas précis au possible inventé. Ce passage résume en fait l'activité mimétique et, selon qu'il ait lieu ou non, sert à caractériser deux types de discours distincts : la chronique qui expose les faits rivés au réel et la poésie qui représente l'homme en action selon une certaine logique du langage. Distinction d'autant plus intéressante qu'elle marque la frontière aristotélicienne entre fiction et non-fiction. Le poète, selon Aristote, ne s'occupe pas du réel mais du possible : le monde vu à distance, donné à voir figurément comme une métaphore du réel. « (...) Il ressort clairement, écrit Aristote, que le rôle du poète est de dire non pas ce qui a lieu réellement, mais ce qui pourrait avoir lieu dans l'ordre du vraisemblable et du nécessaire ${ }^{16}$. Or, la

14. P. Ricceur, op. cit., p. 70.

15. ARISTOTE, op. cit., p. 65 . «(...) La poésie traite plutôt du général, écrit-il, la chronique du particulier "

16. lbid, p. 65. 
mimèsis, semble-t-il, c'est le réel devenu fiction selon une rhétorique et une idéologie que théorise justement $L a$ Poétique : c'est-à-dire le langage comme opérateur de réel au sein de la Cité.

La mimèsis théâtrale, dès lors, est moins la reproduction du " réel » qu'un discours sur un « réel » résolument culturel, et voilà pourquoi il convient mieux de parler de représentation du réel que d'imitation du réel. Nous touchons ici à une première définition du réel tel qu'il s'inscrit dans le discours théâtral et, par extension, dans le langage : le « réel » que parle la scène n'est pas une prise sur le vif, mais une image culturelle du monde qui se fait passer pour naturelle. Ainsi, Aristote, après avoir noté que le discours théâtral est d'abord une mise en acte où chaque je-parlant reproduit une réalité agissante ${ }^{17}$, souligne que «les tragiques (...) s'en tiennent aux noms d'hommes réellement attestés ${ }^{18}$ parce que, dit-il, «le possible est persuasif » et que, pour opérer l'ébranlement du spectateur appelé par la catharsis, ils privilégient les «héros qui ont subi ou causé de terribles événements ${ }^{19}$. D'ailleurs, après avoir cité en exemple les noms d'Alcméon, d'Edipe, d'Oreste, de Méléagre, de Thyeste et de Télèphe, le poéticien ajoute que «la tragédie la plus belle du point de vue de l'art ressortit à cette structure-là " ${ }^{20}$. Si le discours théâtral se donne pour «réel» en s'incarnant sur scène, ce « réel » n'est en fait que l'actualisation et, plus précisément, la dramatisation d'un fonds culturel qui identifie la collectivité : le vrai est ici le reconnu, mais dramatisé. La tragédie efficace, «belle» selon la norme aristotélicienne, ne retient du dépôt culturel que les histoires susceptibles de soulever l'émotion du spectateur : le récit est choisi, si l'on peut dire, en fonction de ses

17. Ibid., p. 53. « (...) La représentation est mise en ouvre par les personnages du drame, écrit le poéticien qui le distingue ainsi des autres genres, et n'a pas recours à la narration".

18. Ibid., p. 65

19. Ibid., p. 79

20. Ibid. «J Redfield, rapporte Ricour, observe que les histoires de héros, reçues de la tradition, sont, à la différence des histoires des dieux, des histoires de désastres et de souffrances, parfois surmontées, le plus souvent endurées. Elles ne parlent pas de la fondation des cités, mais de leur destruction» (p.79). Remarque intéressante qui mériterait d'être vérifiée plus profondément à la lumière des analyses de Jean-Pierre Vernant et Pierre Vidal-Naquet pour qui la tragédie, «cherch(ant) pour la première fois à exprimer l'homme dans sa condition d'agent », serait par conséquent l'expression panique d'une nouvelle Cité libérée des dieux. Cf. Mytbe et tragédie en Grèce ancienne, (Paris : Éd. Maspero, 1973.) p. 70. 
vertus paroxystiques. On pourrait, en exagérant à peine, parler de la reviviscence d'une sorte d'inconscient collectif avec lequel, une fois amené à la conscience par la représentation, le spectateur peut entrer en contact: face à face, dira-t-on autrement, entre une culture et un je à laquelle il participe et dans laquelle il se constitue comme citoyen de la Cité. Plus concrètement, il y a dans l'acte même de représenter un certain plaisir à faire voir «sa" peur ou "son» désir au spectateur, à rendre visibles (lisibles) tels éléments refoulés de la culture ; d'où, selon notre lecture de La Poétique, l'interaction nécessaire entre l'activité mimétique et l'effet cathartique comme l'avers et l'envers d'un même travail sur le « réel ». En cela, la scène est loin d'être la copie du « réel » comme s'il y avait une motivation «naturelle» entre le langage qui la traverse et le monde qu'il reproduit, mais elle serait plutôt le lieu du phantasme culturel. Du reste, il est intéressant de noter avec W. Nestle que «la tragédie prend naissance quand on commence à regarder le mythe avec l'ceil du citoyen ${ }^{21}$, c'est-à-dire lorsque le mythe prend le sens d'un récit légendaire et consacre ainsi un discours culturel. Celui-ci serait en quelque sorte une façon phantasmatique de parler le monde dans la mesure où l'emprise du réel est déniée et tire justement sa force de son irréalité : la culture prise dans une structure qui la déréalise tout en la dramatisant.

L'activité mimétique se situe donc à la jonction d'une culture et d'une structure : elle fait signe à un « réel » que la doxa, c'est-àdire l'opinion publique, accepte comme sien (c'est là le culturel tel qu'il s'inscrit dans toute représentation du réel) et elle insère ce «réel» dans une fiction, le prend dans une structure qui vise à le reformuler à distance (elle représente l'homme en action). Ce passage du réel à la fiction s'accomplit ici en vue d'une libération qui, faute de mieux, pourrait bien être la réalisation du phantasme qui consiste à faire passer la vie du "réel» contraignant au "possible» inventé, toujours selon une culture donnée. De plus, s'il ne propose pas une définition précise de la catharsis, Aristote définit la tragédie comme « une représentation (...) qui par la pitié et la frayeur (...) opère l'épuration de ce genre d'émotions » 22 , laissant entendre par là que la catharsis s'inscrit d'emblée dans

21. Cité par J.P. Vernant et P. Vidal-Naquet, op. cit., p. 25.

22. ARISTOTE, op. cit., p. 190. 
l'élaboration de la mimèsis. «Représenter, soulignent $\mathrm{R}$. DupontRoc et J. Lallot, c'est agencer le système des faits; et la frayeur et la pitié sont d'abord des effets dramatiques inhérents à tel ou tel agencement de faits, à telle ou telle séquence d'événements représentés » ${ }^{23}$. Or, la catharsis résulte ainsi d'une structure qu'Aristote veut implacable «car, dit-il, ce dont l'adjonction ou la suppression n'a aucune conséquence visible n'est pas une partie du tout ${ }^{24}$. Inutile de rappeler que, pour Aristote, «la beauté réside (surtout) dans l'ordonnance» ${ }^{25}$. C'est dire que la lexicalisation sur scène "des événements qui inspirent la frayeur et la pitié » est avant tout le résultat "d'un enchaînement causal qui, ajoute Aristote, se produit contre toute attente ${ }^{26}$. Surprendre le spectateur grâce à une organisation infaillible de l'histoire: telle est, selon Aristote, «la plus grande séduction de la tragédie » ${ }^{27}$. Le poéticien désigne les deux parties de l'histoire susceptibles d'intensifier l'émotion du spectateur pour réaliser l'effet cathartique : il s'agit, bien sûr, du coup de théâtre et de la reconnaissance (anagnorisis) exemplifiés par l'adipe-roi de Sophocle ${ }^{28}$, c'est-à-dire du renversement qui inverse l'effet des actions et du passage de l'ignorance à la connaissance. Le renversement s'associe étroitement à la catharsis dans la mesure où il agit comme « la liquidation active de l'opinion erronée » ${ }^{29}$. Nous n'avons pourtant pas affaire à une « élimination

23. Ibid., p. 254 .

24. Ibid., p. 63.

25. Ibid., p. 59.

26. Ibid., p. 67.

27. Ibid, p. 57.

28. Voir le commentaire d'Aristote à la p. 71.

29. Alexandre NiCEv, L'énigme de la catharsis tragique dans Aristote, (Sofia: Éd. de l'Académie bulgare des sciences, 1970.) p. 162. Nicev propose une explication on ne peut plus moralisatrice de la catharsis en l'associant à «l'indignation du spectateur envers le héros, dont la culpabilité est mise à jour» (p. 88), transformant ainsi sa compassion aveugle en condamnation lucide. On pourrait longtemps épiloguer sur l'origine judéo-chrétienne de cette explication moralisante. D'ailleurs, ne serait-ce qu'à titre de comparaison, on pourrait méditer le point de vue extrémiste de Nietzsche qui, dans l'Antéchrist, conclut avec un aphorisme percutant: "Aristote, on le sait, voyait dans la pitié un état morbide et dangereux, et que l'on ferait bien par-ci par-là de soulager avec un purgatif ». (Paris : coll. 10/18, 1975.) p. 15. De plus, dans sa volonté d'opposer le dionysien à l'apollinien, il ajoute ailleurs : «L'acquiescement à la vie, et ce jusque dans ses problèmes les plus éloignés et les plus ardus ; le vouloir-vivre sacrifiant allègrement ses types les plus accomplis à sa propre inépuisable fécondité - c'est tout cela que j'ai appelé dionysien, c'est là que j'ai pressenci une voie d'accès à la psychologie du poète tragique. Ce n'est pas pour se libérer de la terreur et de la pitié, ce n'est pas pour se purifier d'une émotion dangereuse en la faisant se décharger violemment, 
de la doxa $"{ }^{30}$ selon l'expression d'Alexandre Nicev qui explique la catharsis comme une manipulation rhétorique du spectateur "en résultat de laquelle (ce dernier) prend conscience de l'inconsistance de ses opinions " ${ }^{31}$, mais à une organisation causale de l'histoire où le renversement de l'action marque le paroxysme d'une stricte logique narrative et où la fiction entraîne la dénégation du réel : ou encore, pour reprendre la formule mystique de Barthes, «la possession en vue d'une délivrance ${ }^{32}$.

En fait, s'il faut tenter de la définir, la catharsis est le résultat d'une organisation qui réalise une libération: elle fait appel à un "réel » accepté, voire structuré à dessein, tout en le niant. Elle se traduit donc sur le plan mimétique par une économie de moyens employés pour agir sur le récepteur hors des limites du « réel». Ainsi, l'effet cathartique n'est pas à comprendre comme «l'expérience pathologique du spectateur» ${ }^{33}$, mais comme un certain recul par rapport au «réel» qui permet l'infiltration du plaisir esthétique : le réel devenu fiction. Mais, si le plaisir esthétique se substitue à la peine, quelle est cette purgation équivoque, sinon cette libération, dont parle obscurément Aristote ? Peut-être s'agitil tout simplement, et c'est là le sens le plus convaincant que lui ait donné la postérité, du plaisir «freudien» qui consiste à libérer le spectateur du poids du « réel » et à lui faire vivre " par procuration » une sorte d'émotion quintessenciée : représenter impunément ce qui pourrait procurer angoisse ou désir redoutables. La fiction, si elle déréalise le réel, est au théâtre d'autant plus efficace qu'elle le déréalise bic et nunc: pour réelle que soit la pratique théâtrale aux yeux du spectateur, il ne lui dénie pas moins toute insertion dans la réalité. Telle serait donc la spécificité de la tragédie louée par Aristote qui y voit, pour citer $P$. Somville, une « réalité esthétique où la fiction fonde la libération, et où l'assouvissement, également fictif, confirme l'efficacité, bien réelle, des moyens artificiels "mis

ainsi qu'Aristote l'entendait à tort, mais pour, au-delà de la terreur et de la pitié, être soi-même l'éternelle volupté du devenir - cette volupté qui inclut également la volupté d'anéantir... "Ecce homo, (Paris: Gallimard, coll. Idées, 1978.) p. 79.

30. Ibid., p. 96.

31. Ibid., p. 129.

32. BARTHES, op. cit., p. 71

33. ARISTOTE, op. cit., p. 190 
en œuvre" ${ }^{34}$. D'où, il va sans dire, l'importance accordée à l'agencement de l'histoire brute pour en arriver à la fable comme structure narrative de la pièce, faisant ainsi du discours théâtral l'opérateur d'une suspension de la réalité (se «purger» du réel) qui provoque une émotion de nature à remplacer le danger du «réel » par le plaisir du « possible » : pure affaire d'esthétique dont La Poétique constitue le premier ouvrage décisif.

\title{
C. ARTAUD ET BRECHT: EN DEÇÀ ET $A U$-DELÀ DU THÉÂTRE
}

\author{
«Briser le langage pour toucher la vie, c'est faire \\ ou refaire le théâtre". \\ Artaud, Le Théâtre et son double \\ "Tous les arts contribuent au plus grand de tous \\ les arts, l'art de vivre». \\ Brecht, Petit organon pour le théâtre
}

Les entreprises artaudienne et brechtienne, telles qu'elles n'ont cessé de se théoriser au détriment de la pratique ${ }^{35}$, sont les deux gageures de la modernité pour secouer le joug d'une dramaturgie qui se réclame d'Aristote. C'est-à-dire, selon P. Pavis, «une dramaturgie fondée sur l'illusion et l'identification (...) bâtie autour d'un conflit, d'une situation "bloquée" ("nouée") à résoudre» ${ }^{36}$. Témoin, entre autres, le travail scénique de Peter Brook qui monte le Marat-Sade de Peter Weiss en s'inspirant du Théatre et son double, l'engouement théorique de Barthes pour Brecht et la fascination de Julia Kristeva pour l'écriture dissolvante d'Artaud. De fait, les deux dramaturges n'hésitent pas à signaler leur intention de rupture: Artaud déclare que «si nous avons tous fini par considérer le théâtre comme un art inférieur, un moyen de distraction vulgaire (...), c'est qu'on nous a trop dit que c'était (...)

34. Pierre Somvil.Le, Essai sur la « Poétique » d'A ristote, (Paris : Librairie J. Vrin, 1975.) p. 94

35. Inutile de préciser que l'on va s'intéresser ici à la théorie plutôt qu'à la pratique théâtrale, même si l'écriture dramatique et scénique de Brechr a connu une influence particulièrement féconde.

36. Patrice PAvIS, Dictionnaire du théâtre, (Paris : Éd. Sociales, 1980.) p. 43. 
du mensonge et de l'illusion » ${ }^{37}$, et Brecht d'ajouter : «La dramaturgie non-aristotélicienne, quant à elle, ne condense pas les événements pour en faire un destin inéluctable auquel elle livrerait sans recours l'individu, quand bien même il réagirait avec force et beauté : au contraire, elle examine ce "destin" à la loupe et révèle les machinations tout humaines qu'il recouvre ${ }^{38}$. Certes, leur visée première consiste à dégluer le théâtre d'une tradition aristotélicienne qui, arrangée pour faire illusion, ruine d'emblée toute espèce de dialogue scène-salle au profit de l'adhésion du spectateur à une scène logomachique supplantant le monde. Artaud et Brecht dénoncent une telle emprise de la scène sur la salle et promeuvent un théâtre qui substitue une participation active à l'identification passive qu'ils associent au théâtre occidental. D'où, d'ailleurs, l'attrait quasi utopique qu'exerce sur eux le théâtre oriental ${ }^{39}$, et leur souhait de rejeter le théâtre dans la vie pour ainsi le reformuler en fonction d'une vitalité féconde: le plaisir théâtral n'est plus une simple question d'esthétique ravalant le théâtre au rang de divertissement inoffensif, voire inopérant. C'est là, indique Artaud, «l'infirmité spirituelle de l'Occident, qui est le lieu par excellence où l'on a pu confondre l'art avec l'esthétique ${ }^{40}$ : il ne s'agit plus d'engoncer la création théâtrale dans les vertus emmêlées du Vrai(semblable) et du Beau, mais pour l'un «de désaxer le fondement actuel des choses, de changer l'angle de la réalité ${ }^{41}$, et pour l'autre de «ti(rer) le divertissement de la nouvelle activité productive» ${ }^{42}$. Pour divergents que soient leurs programmes, Artaud et Brecht

37. Antonin ARTAUD, Le théâtre et son double, (Paris: Gallimard, coll. Idées, 1964.) p. 116.

38. Bertolt BRECHT, Écrits sur le théâtre, (Paris : I'Arche, 1963.) p. 97

39. D'ailleurs, Georges BANU souligne justement que la réflexion théâtrale du XXe siècle s'est ouverte à l'Orient pour l'incorporer à sa pratique : "Craig rêve de l'Égypte, dit-il, Artaud de Bali, Tairov de ['Inde, Meyerhold, plus en sourdine, du Japon ». Bertolt Brecbt ou le petit contre le grand, (Paris: Éd. Aubier, 1981.) p. 40. Mentionnons également la fascination brechtienne pour le théâtre chinois à partir duquel le dramaturge élabore le célèbre "Verfremdungseffekt». Brecht, ajoute Banu, «était parti en Extrême-Orient pour y chercher des témoins et des alliés dans un combat contre l'esthétique bourgeoise de l'intemporalité, contre le théâtre culinaire, contre Aristote» (p. 10).

40. ARTAUD, op. cit., p. 105

41. ARtAud, «À la grande nuit ou le Bluff surréaliste ", in OEuvres complètes I, (Paris: Gallimard, 1976.) p. 60.

42. BRECHT, Petit organon pour le thêâtre, (Paris: l'Arche, 1978.) p. 31. 
relancent donc dans le contexte de la modernité la question fort débattue du rapport de la scène au réel et, corrélativement, de l'effet à produire sur le spectateur. Remise en question totalisante qui touche ici à la finalité essentielle de l'œuvre théâtrale et qui réinterprète la relation mimèsis-catharsis, non pas pour la subvertir indûment, mais pour l'assujettir à un idéal. Nous faisons évidemment allusion à l'idéal métaphysique d'Artaud et à l'idéal politique de Brecht qui sont à l'origine même des deux systèmes dont l'un recherche l'insertion de l'être dans le monde et l'autre l'intégration de l'homme dans le social, soit deux directions apparemment opposées : en deçà du théâtre, hors d'une civilisation gangrenée, où tout reste à faire, et au-delà du théâtre, vers une société à transformer, où tout est à refaire.

Le théâtre selon Artaud n'est pas fait pour décrire l'homme, mais pour constituer «l'être d'homme » ${ }^{43}$ : en cela, renonçant à la simple imitation «culturelle», il renoue avec l'étymologie religieuse du mot création ( «tirer du néant», «donner existence») si l'on donne à ce mot la force originelle d'un retour à la nature et à l'individu réconciliés, à ce moment pré-théâtral où l'être est à constituer plutôt qu'à imiter. Création originelle s'il en fût qui consiste à provoquer la fusion pré-verbale de l'esprit et de la matière, c'est-à-dire d'échapper à la signification réductrice du logos, du mot qui «n'est en somme qu'un aboutissement» ${ }^{44}$, en remontant aux origines de la culture : se situer, écrit Artaud, «au point où le monde devient sonore et résistant en nous, avec les yeux de qui sent en soi se refaire les choses, de qui s'attache et se fixe sur le commencement d'une nouvelle réalité ${ }^{45}$. Le réel artaudien, ainsi redéfini, marque le retour utopique à un monde qui n'est pas encore fixé dans l'idée de civilisation. D'ailleurs, ce qu'Artaud retrouve d'abord dans le peyolt hallucinogène des Tarahumaras, c'est un moyen extrême de se purger ou, pour

43. Guy SCARPETTA, "La dialectique change de matière », in Artaud, (Paris : coll. 10/18, 1973.) p. 285. «Le théâtre, écrit Artaud que l'auteur cite, n’a jamais été fait pour nous décrire l'homme et ce qu'il fait, mais pour nous constituer un être d'homme qui puisse nous permettre d'avancer sur la route, de vivre sans suppurer et sans puer/Et le théâtre est ce pantin dégingandé, qui musique de troncs par bardes métalliques de barbelés nous maintient en état de guerre contre l'homme qui nous corsetait ».

44. ARTAUd, Le théâtre et son double, p. 179.

45. Artaud, "Textes de la Période Surréaliste», in L'Ombilic des Limbes, (Paris: Gallimard, 1954.) p. 175. 
reprendre son expression, de se «laver» de l'idée de civilisation, de culture comme négation de la vie: «Or, dit-il, je n’allais pas au peyolt pour entrer, mais pour sortir... sortir d'un monde faux. Nous vivons sur un odieux atavisme physiologique qui fait que même dans notre corps, et seuls, nous ne sommes plus libres, car cent père-mère ont pensé et vécu pour nous, avant nous, et ce que nous pourrions à un moment donné, à l'âge dit de raison, trouver de nous-mêmes, la religion, le baptême, les sacrements, les rites, l'éducation, l'enseignement, la médecine, la science s'empressent de nous l'enlever. J'allais donc vers le peyolt pour me laver» ${ }^{46}$. C'est justement ce parcours à rebours qu'Artaud désigne dans Le théâtre et son double lorsqu'il fait allusion à une «Parole d'avant les mots ${ }^{47}$ où l'agitation scénique se libère de la hantise du signifié dernier pour se donner à un état de signifiance préverbale.

La mimèsis, dans une telle perspective, n'a plus rien à voir avec le vraisemblable qui gagne la crédibilité, voire l'adhésion du spectateur de théâtre aristotélicien, mais, arrachée au dogmatisme de l'imitation, elle se transforme en une évocation matérielle d'un "réel inutilisé par les hommes de maintenant» ${ }^{48}$ tel que le qualifie Artaud: c'est-à-dire qu'elle procède à la réanimation fictive des forces élémentaires d'une nature faussement et perversement domestiquée par la culture. Artaud fait ainsi de la scène un lieu traversé par le mythe des origines où «l'HOMME est seul, et raclant désespérément la musique de son squelette, sans père, famille, amour, dieu ou société » ${ }^{49}$, aux seules prises avec les forces barbares d'une nature brute. Cette remontée fictive en-deçà du théâtre qui, selon Artaud, s'associe à l'idée de civilisation, correspond donc à une brisure du social ou, plus justement, à une perte du sens (du langage) théocratique, pourrait-on l'appeler, où le monde originel, dépris du logos culturel, rend possible l'insertion de l'être dans un nouveau monde «in-signifié». C'est ainsi qu'il faut

46. ARTAud, «Les Tarahumaras», in CEuvres complètes IX, (Paris: Gallimard, 1971.) p. 48.

47. ARTAUD, Le théâtre et son double, p. 89.

48. Pierre BRUnel, Théâtre et cruauté ou Dionysos profané (Paris : Librairie des Méridiens, 1982.) p. 134. Il cite une lettre du 25 janvier 1936 qu'Artaud envoie à Jean Paulhan pour lui annoncer le titre de son livre: Le théâtre et son double.

49. ARTAUD, «Les Tarahumaras», p. 31. 
comprendre le souhait d'Artaud de «briser le langage ${ }^{50}$ pour déréaliser radicalement le social au profit d'un " réel innomé » ${ }^{51}$ qui échappe à la signification culturelle et qui transforme la scène illusionniste en «un lieu matériel pensant ${ }^{52}$ où, pour parler artaudien, l'être se constitue «avec un bruit de création » ${ }^{53}$.

Cette redéfinition du réel modifie largement le type de relation entre le spectacle et le spectateur: ce dernier n'a plus affaire à une scène axée sur l'identification, mais à l'inscription sur scène d'un fonds pulsionnel en vue d'une totale libération culturelle. Il s'agit moins de purger le spectateur de certaines toxines indésirables, ou d'une épuration morale passant par l'écoute du corps, que de le traumatiser métbodiquement, selon une nécessaire rigueur qu'Artaud croit trouver dans le théâtre balinais, en essayant de matérialiser l'inconscient invisible. Ce qu'Artaud propose c'est plutôt de toucher à la cruauté qui, plus qu'un simple élément d'une thématique théâtrale, prend ici un sens tout à fait particulier : c'est-à-dire de communiquer implacablement avec les forces vitales qui, dit-il, «(sont) à la base de la réalité » ${ }^{54}$ et qui, comme les rites hallucinogènes des Tarahumaras, entraînent la déculturation de l'être au point qu' « on ne comprend plus le monde que l'on vient de quitter $"$ \$5. À la notion de catharsis aristotélicienne, Artaud substitue l'idée de «curation cruelle » ${ }^{56}$ qui, en termes plus abstraits, correspond à une traversée hors du logos vers l'origine pré-réfléchie du monde: soit hors du langage par lequel l'être s'est faussement inventé. Langage dont le seul usage désormais, écrit Artaud, est de servir de " moyen de folie, d'élimination de la pensée, de rupture » 57 : de rejet. C'est ce que Kristeva a appelé l' «oralisation du rejet » 58 , c'est-à-dire le refus du texte préétabli au profit du seul déplacement

50. ARTAUD, Le théatre et son double, p. 17.

51. Nous empruntons cette expression à SCARPETTA, op. cit., p. 273.

52. Jacques Garelli, ARTAUD et la création du lieu, (Paris: José Corti, 1982.) p. 87

53. ARTAUD, «Manifeste en langage clair ", in L'Ombilic des Limbes, p. 192.

54. BRunEL, op. cit., p. 18. «(...) Le théâtre dans la mesure où il cesse d'être un jeu d'art gratuit, où il redevient actif et retrouve sa liaison avec des forces, écrit Artaud, reprend son caractère dangereux et magique, et s'identifie avec cette sorte de cruauté vitale, qui est à la base de la réalité ».

55. ARTAUD, «Les Tarahumaras », p. 32.

56. Nous empruntons cette expression à $\mathrm{P}$. Brunel qui entend signifier par là une variante moderne de la catharsis.

57. ARTAUD, “À table», in CEuvres complètes I, p. 34.

58. Julia Kristeva, «Le sujet en procès», in Polylogue, (Paris : Éd. du Seuil, 1977.) p. 97 
de l'air, d'une performance sonore qui échappe à la signification. Scarpetta, quant à lui, parlera d' « une pratique insurrectionnelle du langage » ${ }^{59}$. La «curation cruelle » est donc une sorte d'ébranlement ontologique où c'est « l'idée d'homme » qui est à constituer, tout comme la scène artaudienne "refait poétiquement le trajet qui a abouti à la création du langage ${ }^{60}$. En tant que produits culturels, l'être et le langage sont inacceptables chez Artaud. Celui-ci rêve plutôt à un être in-civilisé, sans langage, pur dirait-il, qui doit «reconstitue(r) l'union de la pensée, du geste, de l'acte " ${ }^{61}$ : le faire et le dire indissociés dans l'être. Dès lors, il appert que «l'anticatharsis » artaudienne, telle que désignée par Franco Tonelli qui y voit « une mise en liberté de toutes les forces vitales de l'homme » 62 plutôt qu' « un processus rationnel (...) s'appuyant sur des émotions de base comme la crainte et la pitié ${ }^{63}$, renvoie (paradoxalement) à une régression en-deçà de l'idée même de théâtre où le jeu est acte vital, constitutif, au lieu d'être une simulation sociale. Régression d'autant plus évidente qu'elle signifie une absence d'être, un retour à la pureté d'avant la venue du sens, qui fonde à la fois le projet d'Artaud et la remarque, tirée de sa réflexion sur le suicide, où il avoue qu' « (il) ne se sen ( $\mathrm{t}$ ) pas l'appétit de la mort, qu'(il) se sen(t) l'appétit de ne pas être " ${ }^{64}$ : c'est-à-dire être là où la pensée et le corps se (re)font mutuellement, là où le corps est «pensant».

L'idéal brechtien, contrairement à celui d'Artaud, ne propose pas une quête des origines, mais s'occupe de l'actualité : d'une apparente régression, on passe ici à une progression vers le social démystifié. C'est-à-dire, sur le plan théorique, dépris d'une supposée immuabilité qui donne la société pour une entité profondément permanente, et engagé dans la voie d'un développement qui fait de l'homme et du monde des produits infiniment «transformable(s) » ${ }^{65}$. L'enjeu de la pratique brechtienne, c'est

59. SCARPETTA, op. cit., p. 272

60. ARTAUD, Le théâtre et son double, p. 167

61. Cité par P. Brunel, op. cit., p. 19.

62. Franco Tonell, L'esthétique de la cruauté, (Paris: Éd. Nizet, 1972.) p. 19

63. Ibid., p. 43.

64. ARTAUD, "Enquête - Le suicide est-il une solution?", in Ceuvres complètes I, pp. 2021.

65. BRECHT, Écrits sur le théâtre, p. 343. «Mais il est une chose qui ne fait plus aucun doute pour moi, écrit-il : le monde d'aujourd'hui ne peut être décric aux hommes d'aujourd'hui que s'il leur est présenté comme transformable». 
justement d'apprendre aux spectateurs à se saisir en tant qu'objets historiques tout comme les personnages mis en scène sont pris dans la texture de l'histoire dont ils ne sont que les objets ballotés: il ne s'agit plus de se livrer au fatum qui légifère les personnages par le relais anesthésiant de l'identification, mais de réfléchir sur les situations qui les agissent. Brecht dit explicitement que le spectateur «ne doi(t) pas simplement (se) mettre à (la) place (du personnage), (mais qu'il) doi(t) prendre position face à lui ${ }^{66}$ : soit se situer face au spectacle plutôt que d'y adhérer inconditionnellement. Nous avons affaire à une scène qui, par les procédés d'étrangéisation de la fable et de distanciation du jeu que Brecht élabore en s'inspirant du théâtre chinois, opère une mise en accusation de l'illusion en instaurant une pratique consciente des signifiants scéniques. Le réel brechtien n'est donc ni le vraisemblable a-historique d'Aristote ni la mise en place de la déculturation rêvée par Artaud, mais, pour reprendre l'expression heureuse de Anne Ubersfeld, « un réel qui fait signe " ${ }^{67}$, si l'on comprend par là que le signe théâtral se construit à partir du réel plutôt qu'il ne l'exprime platement. Brecht renie vigoureusement le «comme si » illusionniste de la mimèsis aristotélicienne et fait du réel scénique un lieu de production signifiant : le réel est fonction d'impératifs idéologiques qui refusent d' "échanger un monde plein de contradictions contre un monde harmonieux " ${ }^{68}$. C'est d'ailleurs dans le caractère résolument adaptable, voire démontable, des divers signes mis en jeu sur la scène que résident la fortune et l'efficacité du théâtre brechtien pour lequel la mobilité du signifiant semble se porter garant d'une transformation sociale à venir, le libérant ainsi du seul critère de vérité générale que sonde le théâtre aristotélicien comme signifié ultime. Soucieux d'échapper au mouvement qui va de soi, à l'autorité de La Poétique, outre l'usage constant qu'il fait de la rupture pour tenir l'illusion théâtrale à distance, Brecht accuse le caractère transformable du réel en le « sémiologisant » de façon à montrer que la seule motivation qui existe entre la scène et le monde résulte d'un choix réfléchi, et non d'un lien naturel qui arrêterait le jeu des signes à l'idée d'une permanence légitimant le mythe de l'éternel humain.

66. BRECHT, Petit organon pour le théâtre, p. 62.

67. A. UBERSFELD, L'école du spectateur, p. 39.

68. BRECHT, Petit organon pour le théâtre, p. 42. 
La scène brechtienne, parce qu'elle est le résultat d'une théorisation qui redéfinit le réel en fonction d'une analyse nouvelle de la société visant à « montrer les événements et les hommes sous leur aspect historique, éphémère ${ }^{69}$, est une pratique qui affiche sa matérialité pour «bloquer» l'illusion mimétique et décentrer, voire décentraliser, le rapport scène-salle. D'où le fait que l'on reconnaisse souvent dans le théâtre de Brecht un théâtre de signifiants : c'est-à-dire un théâtre où les signifiants ne sont pas forcément emportés vers une véridicité fabriquée, mais ouvrent le dialogue entre le monde signifié et le monde réel, faisant de l'écart entre les deux un lieu de production. On sait, du reste, avec quelle verve Brecht s'est opposé au théâtre aristotélicien, en l'assimilant un peu rapidement, comme le note Patrice Pavis ${ }^{70}$, à la seule activité cathartique. Cette réduction de La Poétique à l'idée d'une purgation, si indéfinie soit-elle, témoigne d'un programme radicalement différent : il ne s'agit plus de «sidérer » le spectateur, mais de le «faire penser». Réajustant le plaisir théâtral à son idéal politique, Brecht associe à l'évocation possible des phantasmes la part de réflexion que permet la circulation scène-salle, une fois empêchée la communication unilatérale propre au théâtre illusionniste où l'intérêt de la salle s'englue à la scène. Il va sans dire, par conséquent, que le théâtre brechtien n'est pas « scénocratique » ${ }^{71}$, imposant le langage de la scène comme le seul langage réel, dans la mesure où il renonce volontiers à "la "sidération" du spectateur, et l'oblige à quitter non seulement l'action, la suite du récit, mais l'univers du théâtre, pour revenir à son monde à lui " ${ }^{72}$. Ainsi, la communication envisagée par Brecht ouvre une faille pour avoir lieu quelque part entre la scène et la salle, entre le lieu de production du sens et celui de la réception du sens, dans la rencontre de la fiction (du réel signifié) et du réel (du monde réel) :

69. Bernard DORT, Lecture de Brecht, (Paris : Éd. du Seuil, 1960.) p. 109.

70. P. PAvis, op. cit., p. 43.

71. Ibid., p. 357. «La conception scénocratique du théâtre marque l'aboutissement de la tradition occidentale (incarnée dans l'esthétique hégélienne) : la scène dévoile une vérité profonde du texte et est l'image même de cette révélation du sens. L'espace scénique est sacré dans la mesure où l'esprit d'une cuvre ou d'une époque s'y manifeste. À l'esthétique scénocratique s'opposera ensuite l'idée que la scène fait travailler le sens textuel, le tourne et le retourne, éliminant toute prééminence de l'un sur l'autre, et faisant éclater les limites harmonieuses de la représentation classique».

72. A. UbERSFELD, Lire le théâtre, p. 230 
soit, du point de vue du spectateur, une prise en charge du monde qui marque l'éveil de la conscience politique. Tel pourrait bien être le but de la pratique brechtienne et, par extension, son substitut à la catharsis aristotélicienne et à la déculturation artaudienne : faire de la transformation de la société un «acte de libération » ${ }^{73}$, où, audelà du théâtre, le spectacle se prolonge dans un monde solidaire parce que chaque spectateur devient à son tour, selon son sens étymologique, un acteur, celui qui agit et non plus celui qui est agi.

\section{LA FICTION THÉÂTRALE : \\ UNE DÉPENSE SANS RISQUE}

Il semble juste de conclure que l'idée de catharsis n'est possible que dans le contexte de la fiction: elle implique une identification à la scène qui tient le réel à distance. Il ne s'agit peutêtre pas seulement de la distance entre réel et fiction mais, plus profondément, entre monde et langage (la nomination du monde) : le langage comme loi, coutume, usage, à partir duquel le réel doit se régler. Soit le réel comme langage et, par extension, comme effet de culture. Le réel n'est jamais rien d'autre qu'une formulation culturelle qui le commande et, par conséquent, comme le remarque P. Pavis, «s'identifier c'est toujours se laisser impressionner par l'"évidence" sournoise d'une idéologie » ${ }^{74}$. Qu'il s'agisse de l'obéissance aristotélicienne à une stricte logique causale - laquelle, rappelons-le, traduit la conception du langage comme après coup organisé de la pensée (culture) « déjà là »-, de l'utopique déculturation artaudienne qui vise à faire remonter la fiction jusqu'à sa perte dans l'acte vital, ou encore de l'engagement brechtien qui, plutôt que de montrer la part du réel dans la fiction, s'intéresse aux prolongements de la fiction dans le réel, il y a là trois orientations différentes dans un réel à chaque fois redéfini : transformation, par l'ordonnance du langage, de la «culture» préexistante en "fable» structurée, dépossession de l'idée de culture (comme traînée de langage) par un éclatement exacerbé des langages scéniques, historicisation de la scène repensée comme produit

73. BRECHT, Écrits sur le théâtre, p. 97. "Tout comme la transformation de la nature, ditil, la transformation de la société est un acte de libération; ce sont les joies de la libération que devrait nous faire connaître le théâtre de l'ère scientifique ».

74. P. PAvis, op. cit., p. 209. 
«transformable» en regard de l'amélioration du monde. Nul doute, alors, que la fiction, d'autant plus puissante au théâtre qu'elle se réalise hic et nunc, reste toujours une façon de penser le monde et de le dire : de le parler à l'intérieur d'un autre discours, plus grand, celui de la Culture où le réel (si subversif soit-il) prend racine et s'innocente.

Et c'est là que réside sans doute le plus grand plaisir théâtral tel que l'ont défini les trois théoriciens qui ont retenu notre attention: dans la fonction dé-réalisante de la fiction. «Le plaisir théâtral (...), comme le dit pertinemment A. Ubersfeld, est donc l'union de tous les éléments affectifs, plus l'éloignement qui permet la paix ${ }^{75}$, soit le réglage d'une fiction dont la matérialité est bel et bien réelle. Il s'agit, en quelque sorte, de flirter avec le « possible » figuré sur scène tout en se sachant séparé (protégé) de lui par la rampe, sinon par l'espace du jeu: une dépense sans risque, dira-t-on autrement. Délivrance quelque peu mystique chez Aristote, emportement hors du langage vers l'être originel chez Artaud et volonté de ré-investir le monde à neuf chez Brecht, mais dans les trois cas une même absence de risque indissociable de la carharsis dans la mesure où le monde dont elle nous «purge » n'est pas effectif. Telle serait la spécificité, voire l'impudeur, de l'acte de montrer à l'origine du projet théâtral : faire de la scène un lieu où l'on peut mentir impunément pour dire à « haute voix» le désir «silencieux».

Alain Bernard Marchand, Ottawa.

75. A. UBersfeld, L'école du spectateur, p. 342. 